Groffynld Gphraim Lelfing

\title{
fämflut the
}

Bmeiundzุamąigfter Band

Grtter Geil. 



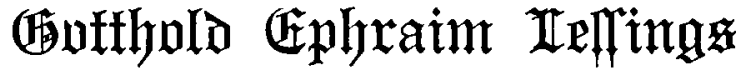

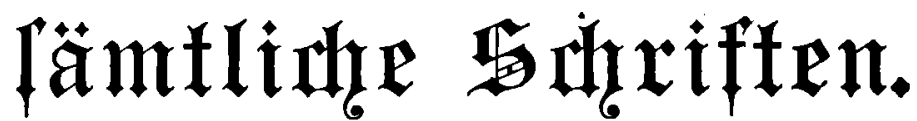

Eุeraungegebru you

\section{值 $\mathfrak{a} \mathfrak{I} \mathfrak{a} \boldsymbol{m} \mathfrak{m} \mathfrak{n} \mathfrak{n}$.}

\author{
Driffe, auf neue Durd)ge[eljene und vermeljrte Tuflage, \\ beTorgf Durdh \\ Frant 142undrex.
}

Bmeitudąuanzigfter Band

Giffer Util.

Betlin und Iriptig.

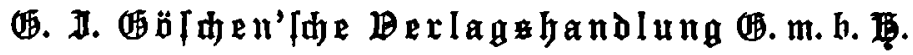
1915. 
Arte Redite oon ber Berlagahanblung vorbehalten.

Drud bon earl Rembolb, Seifbronna. $R$. 


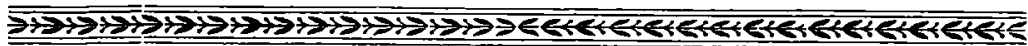

\section{Burrede.}

Der żvciundzwanzigfte $\mathfrak{B a n d}$ meiner $\mathfrak{A}$ uşgabe, für bie Beridjtigungen

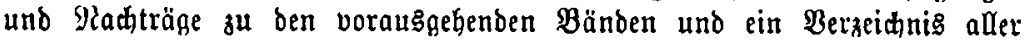

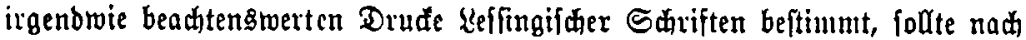
bem urfprünglichen $\mathfrak{B l a n}$ ungeteilt erft in einigen Monaten erfcheinen. Der ungeabnt grobe llmfang aber, den bas Berzeidunis diefer Druffe allmählich gewann, legte ben Bebanten nabe, ben $B$ and in zwei bälften zu zerfdneiden und mit ber erften, die längit fertig vorliegt, fogleid vor bie S̈ffentlichleit ou treten.

J山 ftelle barin zunächit auf lvenigen Blättern bie Sinzeidnungen Seffings in Stammbücher, foweit fie mir belannt geworden find, möglidft

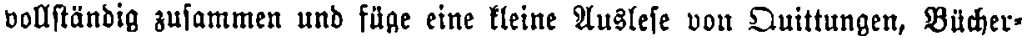
faheinen unb ähnlichen Betteln bei, um bann über breibundert Seiten mit

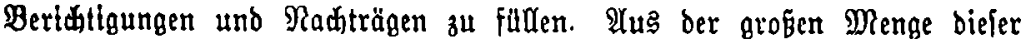
Berbefferungen und Errgänzungen bitte ich aber nidft auf einen bejonbern Peidtum an Feblern in ben frübern Bänden fallęenen zu wollen. Biveifellos finden fich in ignen mebrere Jrrtilmer, bie id nun naw beftem Wiffen und Bewiffen zu beridhtigen fuchte, und ztwar, mie fich von felbit verfteft, ohne Unter/hied, ob midy auf den Fehler ein freundicher Berater oder ein gefäffiger Rörgler aufmerkfam gentacht batte. Selbft bem roiderlich anmabenden Budbe

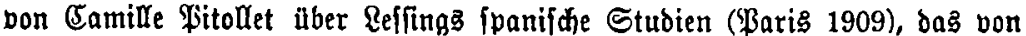
unbegrünbeten 2lngriffen auf beutide Belehrte wie auf Reffing felber ftrozt und jeben $\mathfrak{U}$ itand gegen frembe Foriajer vermiffen läbt, fonnte iø einige braubbare Belehrungen entnefmen; fein fonftiges grobes Bepolter und wiffen= (ihaftlith wertlofes (Serede ift leiner Wiberlegung wert. Für afle diefe Beridtigungen aber bätte ein Drudfbogen ober rveniger reid)lid genügt. Die

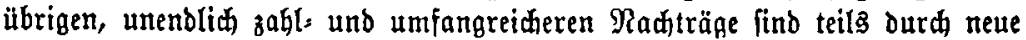
Funbe Reffingifder Sandfariften, von benen id früber noh nidita wiffen

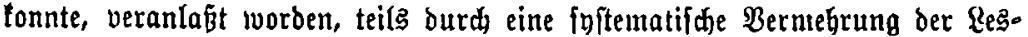

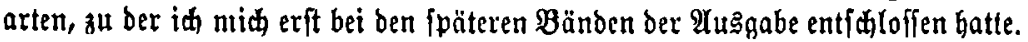




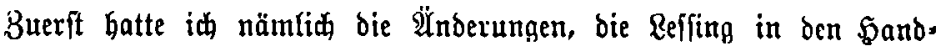
fahriften noh vor bem Druct gleich während bes Shreibens vornahm, grund. fäblich nicht mitberzeidanet; erît von Band XIII an tat idh es regelmäbig.

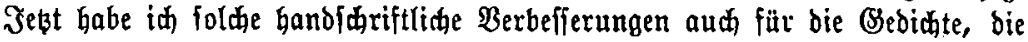
Dramen und bramatifhen Entwürife und ben "Eaofoon" nadgetragen. Seben ifnen mertte ich jeşt aud noh mandes andere an, twas ich zubor abfichtlich

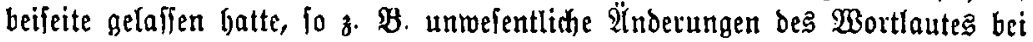
Büfnenamweifungen und unter llmfländen au山 unztweifelbafte Drưffebler, bie

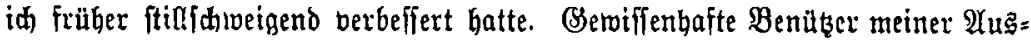

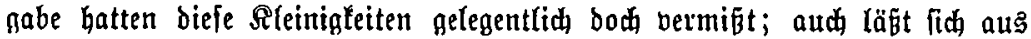

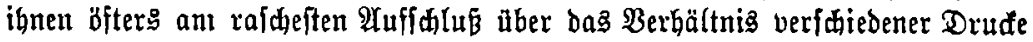

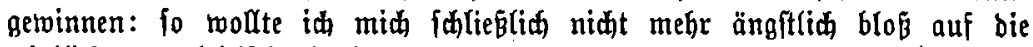

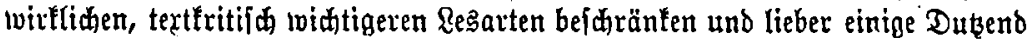

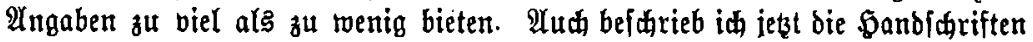
felbft, foweit ich fie zu erneuter Bergleichung einfeben fonnte, viel genauer als zuvor und bruate namentlich bie (Entwürfe sum "Satban" aud äuвer= (id) möplichit getreu nach den Driginalpapieren ab. Die flüchtigen, mitunter faum lesbaren Shriftzïge Reffings habe ich ftets zu wieberbolten Malen auf

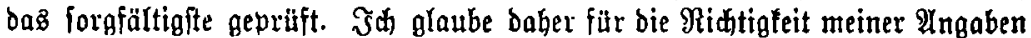
bcionders auch in jenen - nidbt allzu bäufigen - Fällen einftelgn zu fönnen,

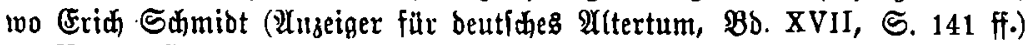
im Rathan=Entwurf ober in ber "Matrone von Ephefus" sine andere, bisweilen fogar verführerifder faheinende Refung voridhlug.

Sonjt vermebrte in bie in Band III abgedrudten bramatifuen Ent. woürfe burch bie l̈beriebungăbruffitüte aus Thomjons Traueripielen und ben rätfelbaften cinatter "Borabe", ben idh zrwar auth jest nidht für ein Werł Reffings balte, aber gegenulber ben zuberfichtlicheren Bermutungen andrex

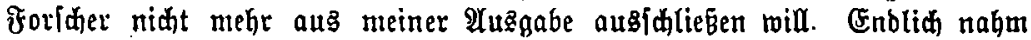

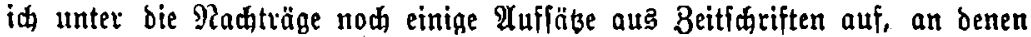
ber junge Reffing Intcil Gatte, ba neuere Unterfuchungen feine lurbeberfdaft für diefe Beiträge utebr ober weniger mabrifheinlid gemadit baben.

unter ben neuen handfhriftlichen Funden find die 2Ynmertungen zu

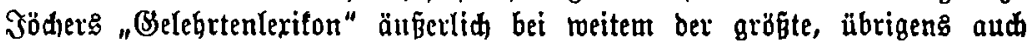
für Eeffings gelchrtes Wiffen und Freube an gelebrter Rleinarbeit bezeidfunend. YYn fie reigen fĭ, geringer an $\mathfrak{U m}$ mang und Bedeutung, bie Bemerfungen zu

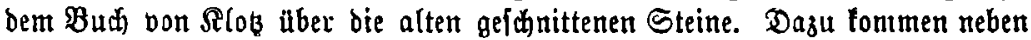

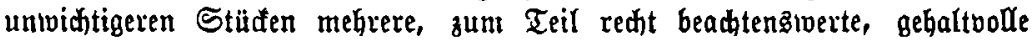
Briefe von Reffing, aud einer an inn. (Enblid teile if eine darafteriftifase

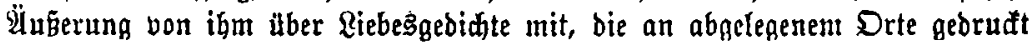
ift und beshalb biaker ftets itherfehen wurbe.

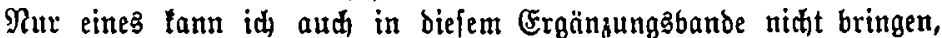
bas freilith zur unbedingten Bouftändigleit ber Squiften Reffingz gebörte, bie Überleţungen gröberer Werte, die er befonbers in jungen Jahren, gum 


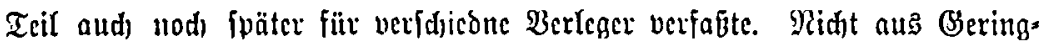

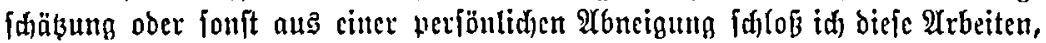
die ia meiftenz nur bez Eriverbs lyalber unternommen und mit geringerer

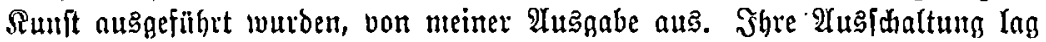

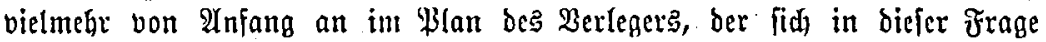

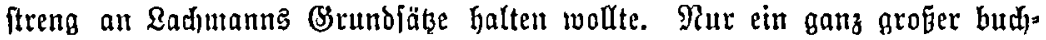
Gändlerifdace Erfolg gätte ifgn von biefer 2lnfidat belegren uno zu ben weiteren, beträdntlidhen Dpfern beftimnten fömmen, bie der Drutf ber lïberfelizungen er=

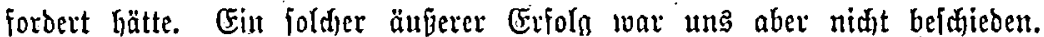

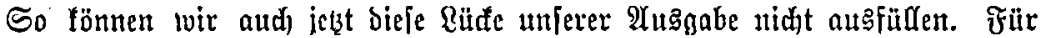

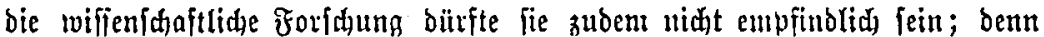
wenigftens die literarifa, bedcutendften libcrtragungen Reffings find ihr burdy

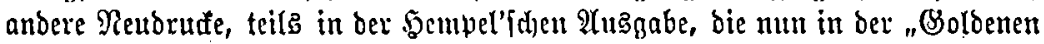

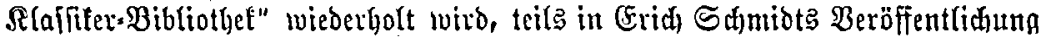
von 1892 , benuem ă ăg̈nglid).

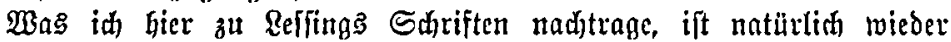

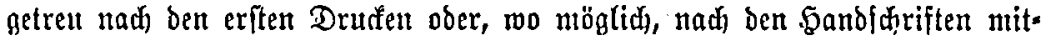

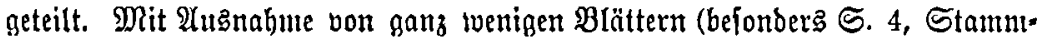
buffblatt 5; 5. 10, Quittung 9; 5.308 f., Brief $\Re \mathfrak{R}$. 714 a und 783 a),

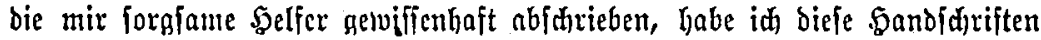

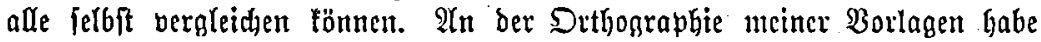

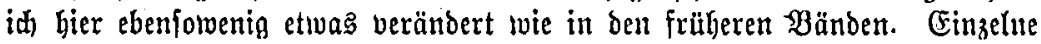

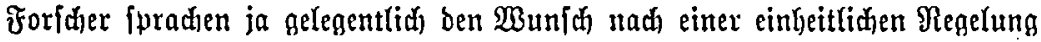

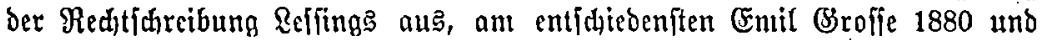

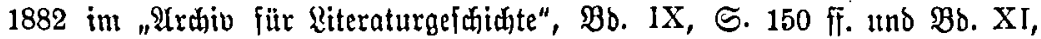
S. 367 ff. Ere meinte, man folle aus ben erbaltenen Sandfdyriften bie

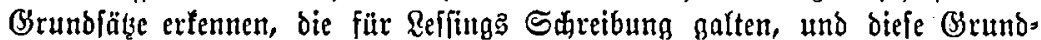

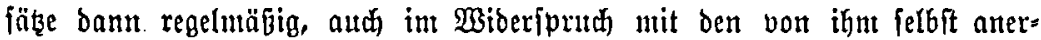

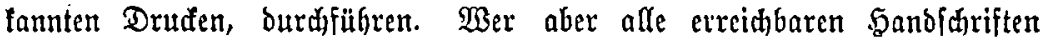

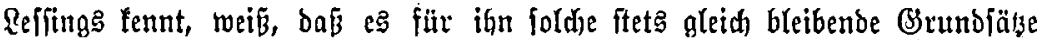

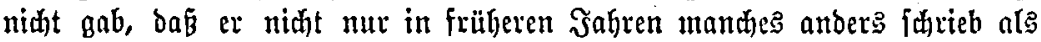

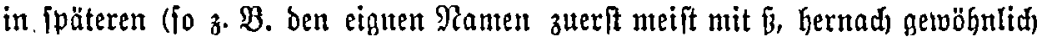

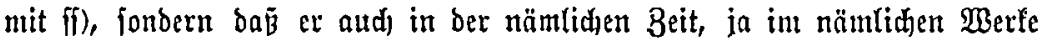
zwifden $\mathfrak{B}$ und ff unt andern ägnlid)en Reteinigleiten oft beliebis toedjfelte, felbft bei ber wiebergolten Sdjreibung ber gleiden Wörter. So müßjte jebe cinfeitlithe Regeltung feiner Srthographie, befleifigigte man fid babei aud ber peinliduften Sorgfalt, nur zu Wialürtidfeiten im cinzelnen füfren. (E) bleibt ba\& einzig Sidjere, fid genau an bie hanbfdrifftliden ober gebrutten Borlagen mit allen ifren Sdiwanlungen zal balten; gerabe in biefen fpiegelt fid Refïngs Bebandlung foldter nebenfädlitłen Dinge an beften ab.

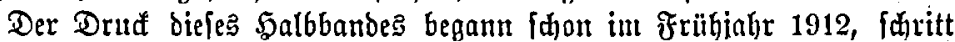

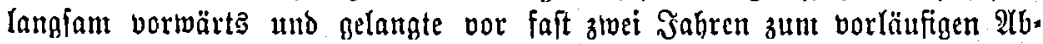

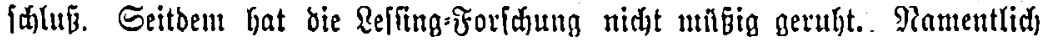




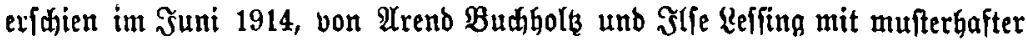

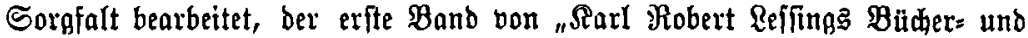
bandfhriftenfamnlung" nit zabłretden, mertvollen Mitteilungen von unge=

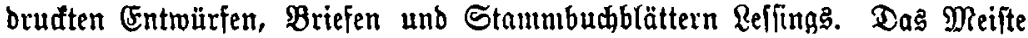

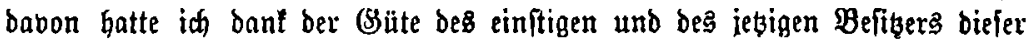

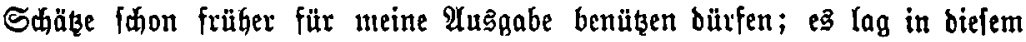
Ergänzungabande längft gebrưđt vor, nur war e马 nod) nidt nuşgegeben worben. Einzelnes aber, was ber Reeffinglatalog entbielt, war auth mir vorber unbelannt geblieben. Diefez unb noch cinige Ergebniffe eigner unb frentber Befđäftigung nit Reffings $\mathfrak{B e r f e n , ~ b i e ~ e r f t ~ b i e ~ L e t ̦ t e n ~ F a b r e ~ g e z e i t i g t ~ b a b e n , ~}$ mödte id gleid bier nađbolen.

Bon Stammbuchblättern Refifings fann iđ außer ben S. 1-8 mitge= teilten now vier weitere anfübren. $\mathfrak{D a s}$ erfte teilte Mar Morriz im Tanuar 1904 in ber Beefelfffaft für beutfde Riteratur zu Berlin mit; darauf

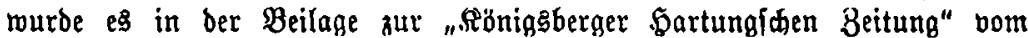

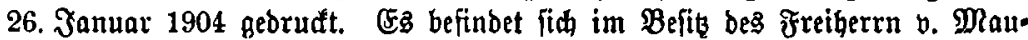

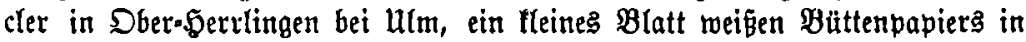
Querottab, nit faubern, beutlidien Bügen bejdrieben, und lautet:

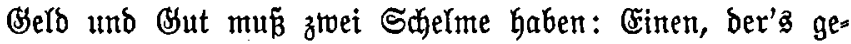
winnt, und Cinen, ber's verthut.

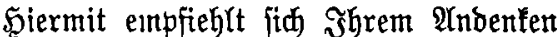

\section{Leipzig}

Den 14. Dec. 1757.

Séping.

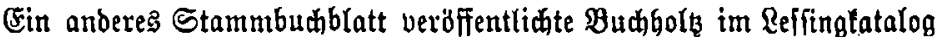

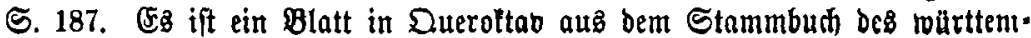

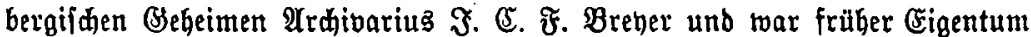
Wendelin v. Maltzahns, bon bem es Rarl Robert Reffing 1886 taufte; jețt

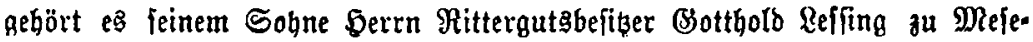
berg bei Berlin. Ess Yautet:

Spe et bona Mente.

im. c. ses.

Gotthold Ephraim Lessing.

Wolffenb. d. 8 Maj. 1770.

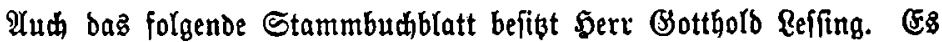
ift ebenfalls ein fleines $\mathfrak{B l a t t}$ in Queroftab, mit fauberen utnd beutlithen

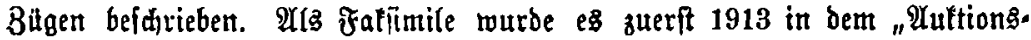

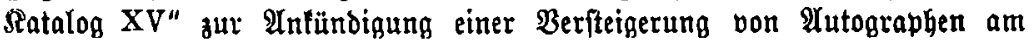

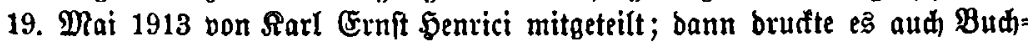
holla a. a. D. S. $188 \mathfrak{a b}$ : 
Fortuna vivat quisque contentus sua.

m. c. scb.

Gotthold Ephraim Lessing

Wolfenbut. d 25 August.

1772

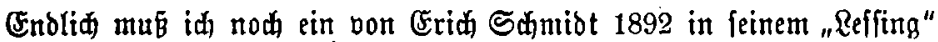

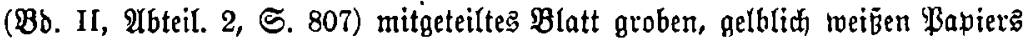
in Dueroftab, mit beutliden Bügen befdrieben, aus bem Befitio Allbert Röfter: nadtragen:

\author{
Ev $\varepsilon \gamma \omega \varkappa \alpha \iota \pi \alpha \nu \tau \alpha !$ \\ (Sotthold Ephraim Reffing. \\ Şamburg \\ Sent 14 Dutbr. 1780.
}

Das S. 5 angefübrte 7. Stanmbuchblatt, deffen gegentwärtigen $B_{e}=$ fiber id bort nidht zu nennen muşte, ift ieșt gleidfalls Eigentum bes Botthold Refing; Buchbolk brưte es a. a. D. S. $188 \mathfrak{a b}$.

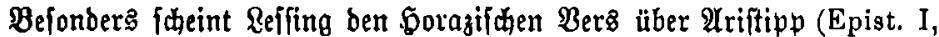
17, 23) geliebt zu haben. (Er verwertete ihn, forweit bisher betannl geworden ift, breimal. Buerft farteb er ifn dem Studenten Johann Beorg Ed, bent fpäteren Reipsiger Brofeffor ber Didfttunft, 1766 in bas Stammbud. Lilber

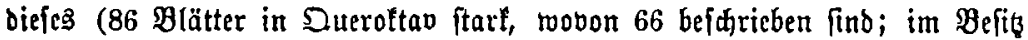
ber Roge "Minerba" zu Reipzig) beridftete augfitbrlid 1879 in ben "(3renz. boten“" (4. Suartal, S. 326-334) ein ungenannter MRitarbeiter und teilte mebiere $\mathfrak{B r o b e n}$ baraus mit, $\mathfrak{S} .333$ auch Reffings Eintrag, den hernadh wieber $\mathfrak{B}$. v. Maltzafn und $\mathfrak{R}$. Borberger 1881 in ber zweiten 2 (uflage von Danzels und Bubrauers "Reffing" (Bo. II, S. 635) abbrutten :

Horat.

Omnis Aristippum decuit color et status et res.

m. c. sc.

Gotthold Ephraim Lessing.

Berol. d. XVI. Aug. 1766.

Dann mieberfolte Refinting biefelbe Einzeifinung 1772 in bem Stantmbuth

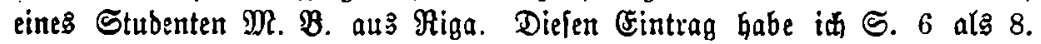
Stammbutbblatt mitgeteitt; er ift jestt auth von Budfolts a. a. D. S. 188 abgebruaft. Bum britten $\mathfrak{M a l}$ verwanbte Reffing ben gleiden $\mathfrak{B}_{\text {er: }} 1774$ für 
Burrede.

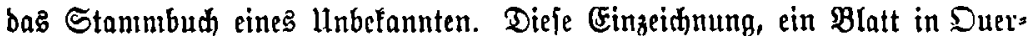

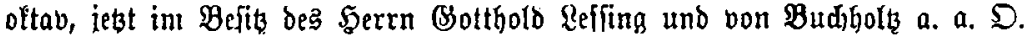

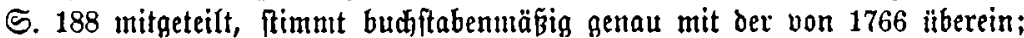
nur fegflt bie lüberidhrift „Horat.“, und bie lek̨te Beile lautet: „Wolfenbüt. d. 4 Maji $1774^{*}$.

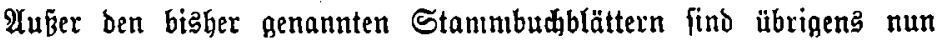

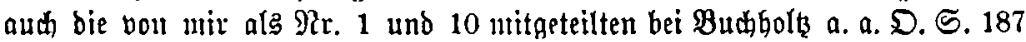

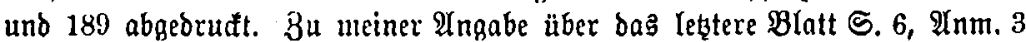

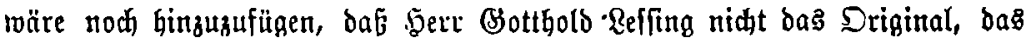
fid int o. Bergmann'idfent Familienardfio zu \$otabam befindet, fondeen nux pin Fraffimile bavon befitit.

2bbfidtlih nidjt aufgenonmen habe id brei Bcilen, bic zuerft 1821 in bent von $\Im$. Wenzel Rembert herausgegebenen Wiener "Tafdenbud filt

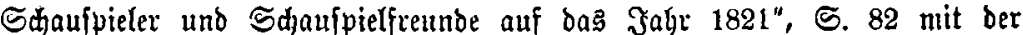

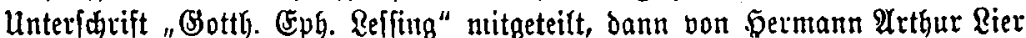

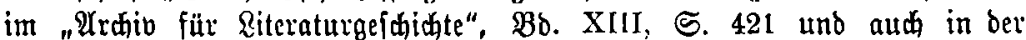
Reffing.2Yuąpabe ber "Bordenen Rlaffiter=Bibliothet", Bb. I, S. 44 wiederholt murben. Sie befinden fid in bent Stanmibud) des Sdjaufpielers Sofann Frana Sieronymug Broffnann (in Sueroltav), bas nah bem Tobe feines

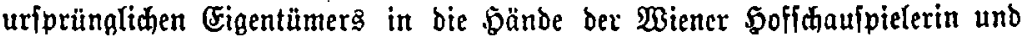

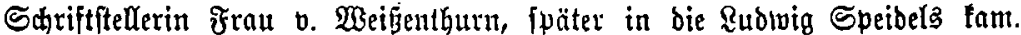
Die Freunblid)feit ber gegenmärtigen Befiţerin Fräulein Reontine Speidel in Wien ermöglidt mir, bas Blatt nach einer genauen Baufe bier mitzuteilen :

Bebfall tann wie Bolb eridiliden werben,

Und Talent erzloinget ifgn:

Ther Aller Betafall fein Talent.

Berlin

ben 24 Merz

Siarl Bsotth. Reping

1778.

Dẩ nidht Botthold Eptraill Reffing, fondern fein Bruber ber Satreiber

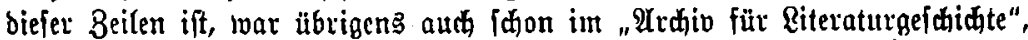

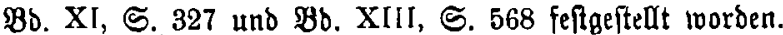

Bon ben Quittungen unb Büheridgeinen hat bie von mir auf S. 10

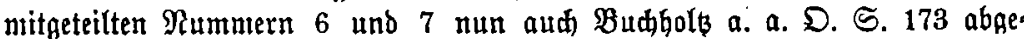
bruatt. Bei $\mathfrak{R}$ r. 6 bätte idh auf $B$ b. XVIII, S. VI verweifen folten, wo idf bie paar Beilen nad) einem nidht budfitäblid gentauen $\mathfrak{Y}$ tboruc in einem antiquarif hen Ratalog beteits nitgeteilt hatte. $\mathfrak{A}$ uf ein weiteres berartigez

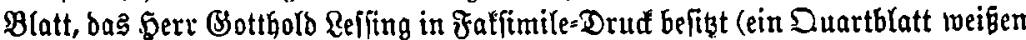
Butttenpapiers, nur auf einer Seite mit beutliden, faubern Bügen befarteben) roeift $\mathfrak{B u d}$ holth an ber gleiden Stelle (S. 172) bint; id Iaffe es bier folgen:

1. Codex mnspt. Fomeri, unter ben Rehdigeriden Msptn nad bem Kranzifhen Cataloge No. VI. in Fol. 
2. Antonii Panormitae Carmina \& Epistolae, unter ben nehm= liden Msptn No. LXXXV. in Fol.

3. Homeri Batrachomyomachia per $\mathrm{C}$. Aretinum carmine latino reddita; it bem Codice No. CLVIII.

4. Senecae Epistolae, No. CXII.

Borftelyente Manuscripte erbittet fich ganz ergebenft auf furze 3eit von ber Bibliothef zul Sิt. Erijabeth

Brestaul Den 10 Decbr. 1763. Bottgold Ephraim Rejiutig.

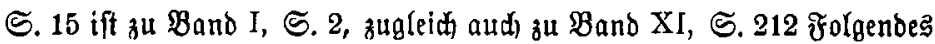
nadızutragen. Der zweite Druff be马 erîten Teils der "Bermildten (Sämtliden) Sthriften", von mil als 1771 b bezeidnet, fann, wie ih erft bei ber 2Uuzarbeitung ber Bibliograpgie mit voller Deutlichteit erfannte, wegen ber

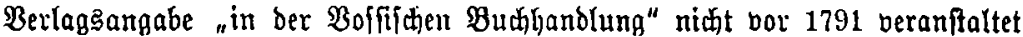

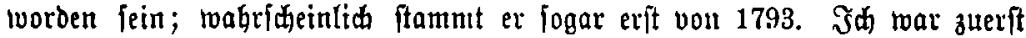

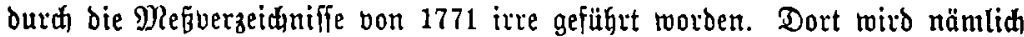
biefer Band jøon zu Sftern und bann wieder zu Michaeliz, jestzt mit bem

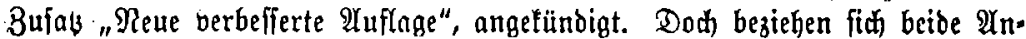
gaben auf ben Druđ 1771 a, ber nad ber (Ertoartung bea Berlegers fafon zu Oftern fertig werben follte, in WBirffichleit aber erft im jerbft erfacinen

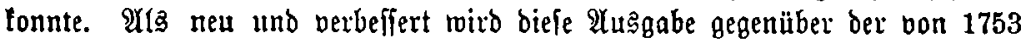
bezeidfnet. Demnach follten aư in den vergältnismäbig feltnen föăllen, two fï bie Druffe a und $\mathbf{b}$ in Wortlaut ber Sinngebidte unb Rieder unterfateiben, in $\mathfrak{B a n d}$ I meiner 2 Uusgabe meiftenz bie Rejarten von 1771 a, bie jeşt unter ben $\mathfrak{A}$ nmerfungen fteren, in den Text, bagegen die Resurten von

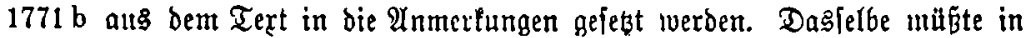
Band XI bei ber 2lbhanblung über bas Epigramm gefdeber. Es hanbelt

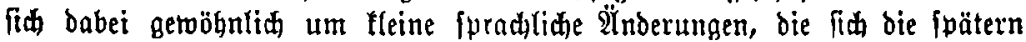
Derausgeber an Reffings Worten erlaubten. Sie und ba berifatigt aber $1771 \mathrm{~b}$ aud einmal einen wirtlidien Fefler von 1771 ; da bürfte natïrlidid eine foldhe Berfodiebung nidit ftattfinden.

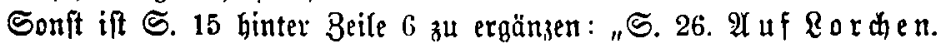

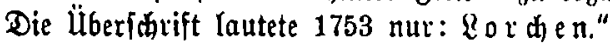

S. 17, 8.15 ift zut Tejen: voin [iftatt "von"] Sertn Boftrath

S. 20. Reben ben Gier befprodhenen unedten Bebihten wäre nod ein

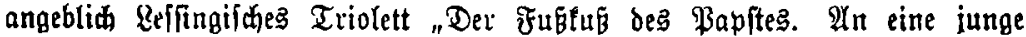
Dame" zu erwägnen, bą ein llngenannter in ber "Mational=Beitung“ vom

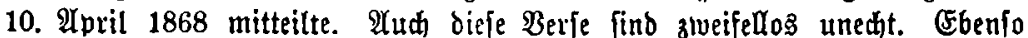
bas angeblich Reffingifdhe Ried "ÎAufntunterung", bas ber britte Teil von Fro. Wilt. Marpurgs "Berlinifaten Soen und Riederu" (Reiwzig 1763) S. 40 entbält; es ftanmt in $\mathfrak{B i r f ( i a d ) l e i t ~ v o n ~ \Im o b a n n ~} \mathfrak{A}$ tnolo Ebert.

S. 21 f. ift zu ben Radträgen zur Doe auf den Tod des Marifaula v. 


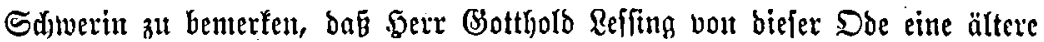

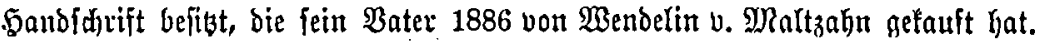
Sie befteht aus einem ₹oliobogen bïnnen 2 ättenpapiers, von bem brei Seiten bejdrieben find, und ift vout $\mathfrak{B} u d$ dyola a. a. D. S. 167-169 mitgeteilt

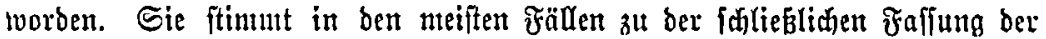

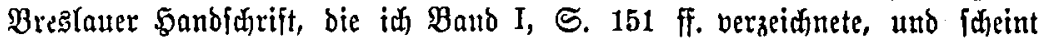

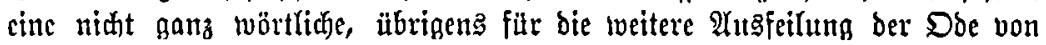

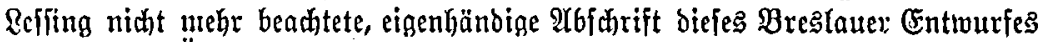

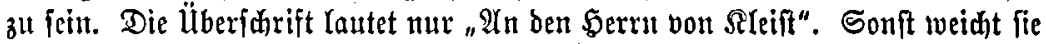

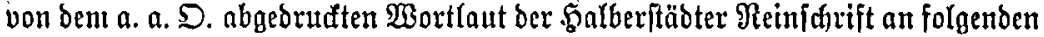
Stellen $\mathfrak{a b}$ : S. 151, 3. 25 viel [feflit] 3. 26 f. zut trennen, Mars bet

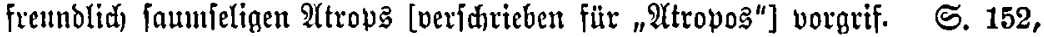
3. 4 f. o Rleift [und] Brar [fegit] 3. 7 ben 3. 11 entlefnt; 3. 34 verbedt [bafinter] bie [burdfittiden] 3.15 Burpur. 3.16 Bezauberung? 3. 17 Sein Sdjüler; fein Märber! 3. 20 Da rollen 3. 21 wäfht 3. 22 abgetwandten 3.25 bie Bewunberung: 8.26 o Siteit, [und] Did

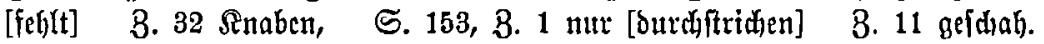
[verbefiert aug] gefdelen 3.14 [Ginter "ntit"] Bar [burdfftriden] B. 19 ff. Der entfäulte ßallajt über Did, Simion, ein fafredfidues Monumtent von Muinen und zerfdhmetterten Žeinden, zufammen; und Dein Tod warb

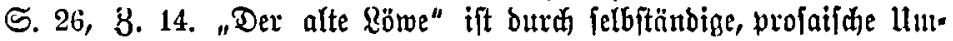
geftaltung einer Frabel BSleims entftanden.

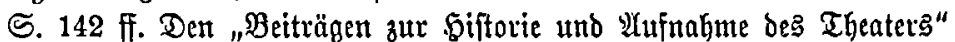

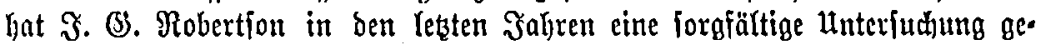
lvibmet (The Modern Language Review, Bb. VIII, Sheft 4 vom Oftober 1913 , ธ. 511-532 und $\mathfrak{B b}$. IX, Seft 2 vout $\mathfrak{2}($ pril 1914, 5. 213-222).

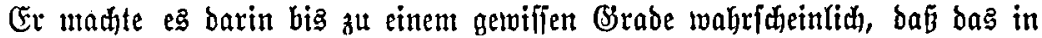
bie "Rritil über bie Befangnen des \$lautug" eingefdhloffene Sdareiben eines

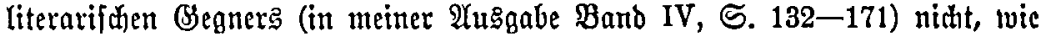
man biabler meinte, von Refing felbft berrühre, ber Gier unter frember Magle jeine lïberfetzung uno fein Reben beg alten Deifhters mit Saheingrünten angreife, fonbern in ber Tat von einent jetzet nidjt mebr genauer feftzuftellenden fremben $\mathfrak{B}$ erfaffer an bie Şerauggeber ber "Beiträge" eingefandt worben fei.

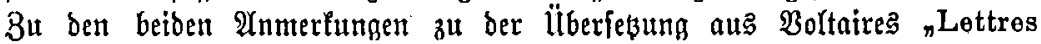
anglaises" nimmt Robertfon ungefäffr bie gleidfe Stellung ein wie id (S. 142). Die "Thbeatralifaten Reuigfeiten aus \$aris" fübrt ex ebenfo wie Beterfen, boch unabjängig bon ifm, auf übertragungen aus ber "Bigarure“

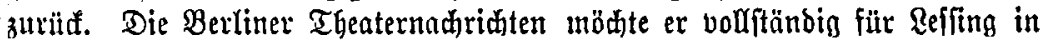

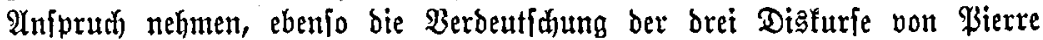

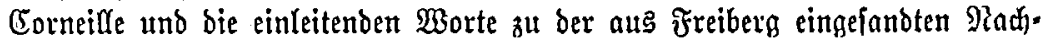

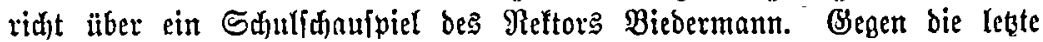

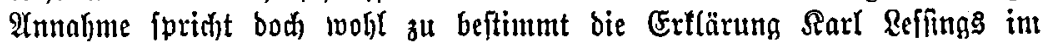

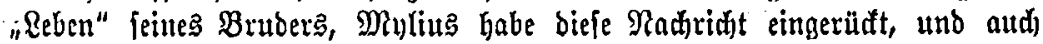




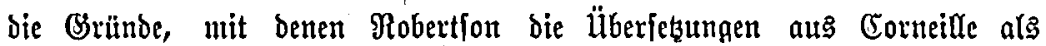

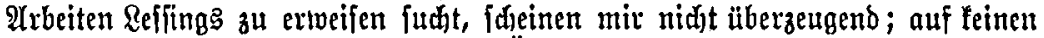

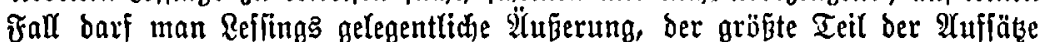
in ben "Beiträgen" fei aus feiner Feder gefloffen, fo budfftäblid verftebn, als ob man ihm nun, nad) Seitenzahłen nusggerednet, minbeftens zwei Drittel ber ganzen Beitidurift mit Bsewalt zujwieben mübte.

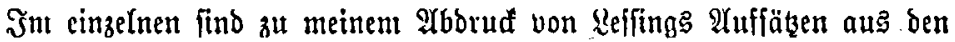
"Beiträgen" in Band IV nod) folgende von Robert[on angeregte Beridti-

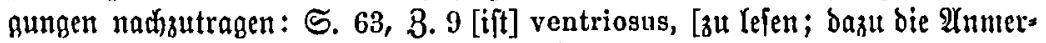

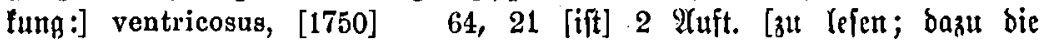

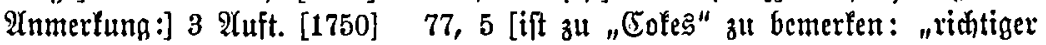

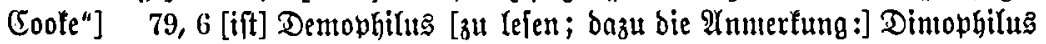
[verbruftt 1750] 80, 7 [ifit] Cieco [ž Lefen; bazu bie 2(nmerfung:] Cicco

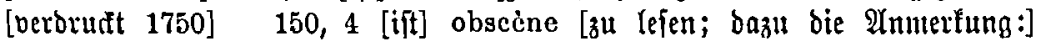
obscure [1750]

S. 157 am Sdyluffe ift beizufügen:

Berlinifde pribilegirte 3 eitung. 1753.

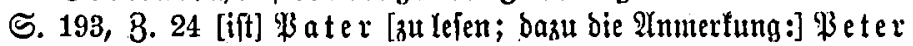
[1753]

S. 161. Die Ergänzungen zu Band VII follten nit folgenden $\mathfrak{B} c^{=}$ ridjtigungen beginnen:

$$
\text { Berlinifue privilegirte Beitung. } 1755 \text {. }
$$

5. 24, 2(nnt. 1 [ift] Donnerftag, [zu lefen ftatt] Dienitag,

ธ.

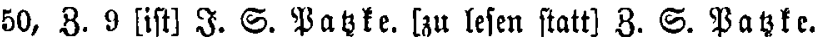

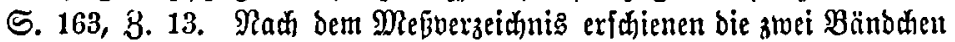
"Die $\mathfrak{B e r r a ̈ t e r " ~ e r f t ~ s u r ~ D f t e r n e f f e ~ 1 7 9 3 . ~ S i e ~ w e r b e n ~ a l f o " ~ a u c h ~ i n ~ b e r ~}$ Bibliographie int zweiten Ieil biefe’ zweiundzwanzigiten $\mathfrak{B a n d e z}$ exft unter bent Jahre 1793 verzeidnet.

ธ. 196, 3. 17 ift "änthlidjen" ftatt "ähnlidfen" zu leien.

S. 200 an Sdfluffe der von Seite 199 berüberreidfenden $\mathfrak{A} n$ merfung, wo auf meinen $\mathfrak{A} u f f a b$ über neue Reffing

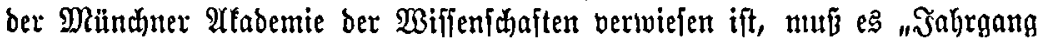
$1915 "$ (ftatt "1913") Yeifien. (Ebenjo 5. 275, 3. 27.

S. 276, 3. 3 ift, worauf mid Gerr Dr. $\mathfrak{T}$. $D$. Ardelis in Bremen aufmerffam uadte, zu ben "Rollettaneen" nod nadjutragen: $5.422, \mathfrak{A n m}$.

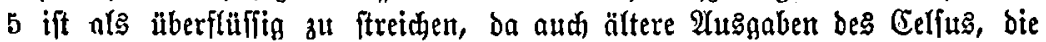
Seffing benittzen fonnte, fo die von $\mathfrak{A}$ Intelobeen (Lugduni Batavorum 1730), „cujusdam" Iejen.

S. 277. Ginter 3.8 wäte zu $\mathfrak{B a n d}$ XVI, S. 96, Atrm. 1 nod ber Sinmeis auf iene Entwürfe zur Sefujihte ber Fabel einzufügen, bie fdon in frïferen $B a ̈ n d e n ~ g e d u u d t$ fino, fo auf $\mathfrak{B a n d}$ XIV, S. 227-244 und Band XV, S. $458-460$. Ebenda follte 孔 
Jahreşzahl 1438 in 1440 zu berbeffern ift. A4ud biefe Belehrung berbante

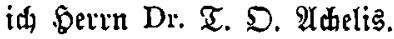

S. 301. Sinter 3. 4. ift Frolgendę zu ergänzen:

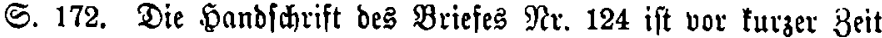

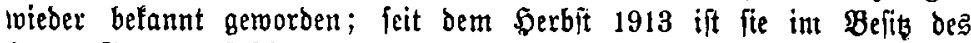
Ferrn Botthold Rejing. (ES ift ein Frolioblatt, bas auf ber Borberfeite den Brief, auf ber SRüuffeite nur bie 2rbreffe enthält. Das von utir S. 172 2rbgedrufte bildot nur ettoa bas leşte Drittel bes Briefes; bie

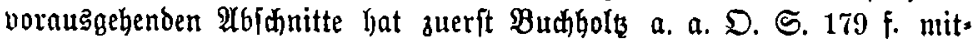
geteilt. Sie lauten:

â Monsieur

Monsieur Lindner

très célébre Recteur de l'Ecole

Cathedrale de $\&$

à

Riga ${ }^{1}$

5odjebelgebohruer 2 .

5odyzuefrender $\mathfrak{p e r r}$,

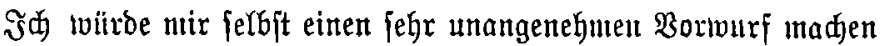

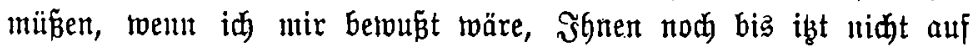
Dero angenefnnez Sdreiben geantivortet za haben, weldjes id be:

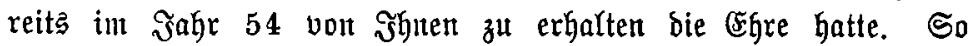

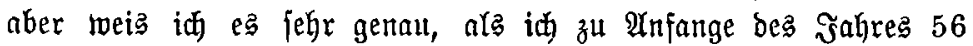
int Begriffe jtand, Deutfdland auf eine geraume Beit zu verlápell,

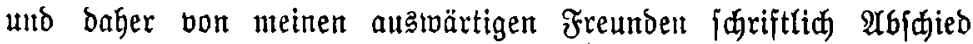

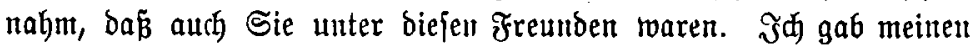

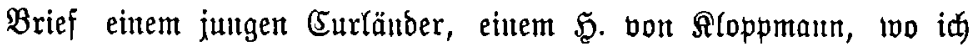
mi出 redft erinnere, bent idj bama(s in Reipzig hatte fennen lernen. Er reifete nad) feinem Baterlanbe zurüa, und verfprach nir, ihn

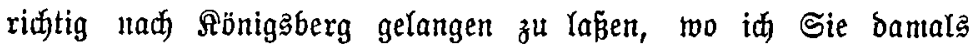
nod) vermuthete. Wern er es nidgt getfan hat, fo betauere idf

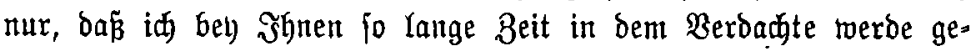
ftanden babelt, idj milïe fo wohl gegen ben Bebfall eilles fäönen

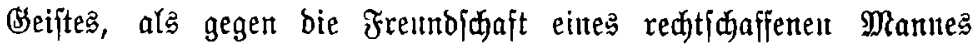
fehr falt gefintut jegn.

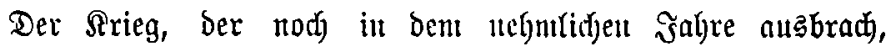

\footnotetext{
[ [Darunter von \{päterer 5xib:] une lettre du famoux Lelsing. grand critiqne et poëte $\dagger$.
} 


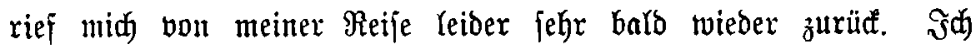

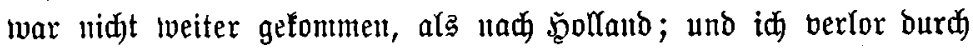
Diejen unfeligen Arieg - (aber taujend andere haben noch weit

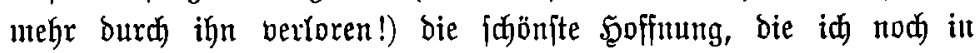

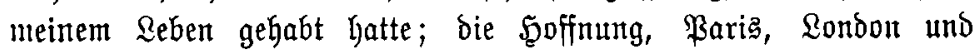

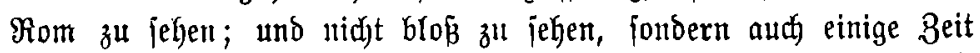

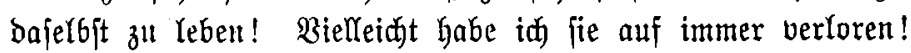

Gier fofliest fith, auf einer neuen Brile beginnend, bas bereits

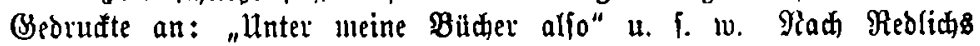
"EeffingsBibliotbel" (Zefitings $\mathfrak{B e r f e , ~ B e r l i n ~ b e i ~ B u f t a b ~ S e m p e l , ~ T e i l ~}$

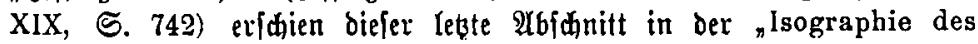
hommes cellebres" fiton 1828 in Band II; dod fonnte ith bisher bie

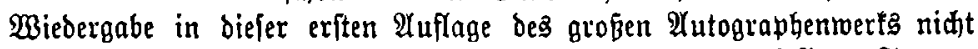
ausfinbig madhen. Freilidy feflen in bem (Exemplar ber biefigen Staats= bibliothef einige Kieferungen; aber biefe gefören bem britten, niwh bem

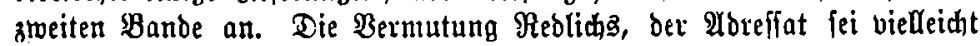

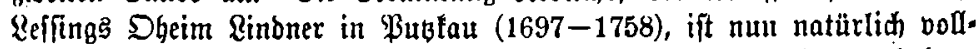
fommen Ginfällig getworden. Sie roar ofynedies nidt mehr zu halten, feitbent man baß Tobesjabr biefes Dheims tannte. Bielmehr ergibt fidh iest aus unferm Briefe, bú Beffing fanon früher mit bem jüngeren

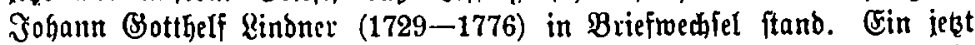
verlorenes Shleciben \&inonets alts Siönigsberg von 1754 wäre unter bie Briefe an Reffing etra ala $\mathfrak{R r}$. 25 a einzureifen, ebenfo bie längit ber:

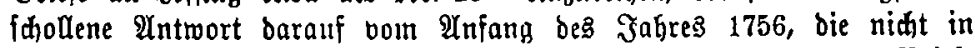
Qinoners Gände gelangt zu fein faheint, etwa alg $\mathfrak{R}$. 43 a unter bie Briefe von Reffing. über ben Эnhalt von Rinbners Sareiben wiffen wir gar

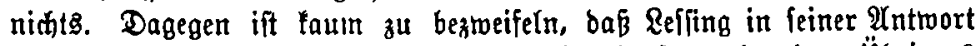

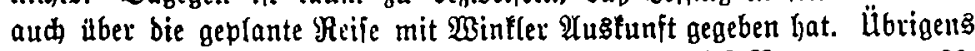

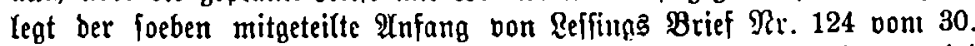
Dezember 1759 bie Bermutung nahe, baß̈ fidh furz vorther Linoner bei ihm beflagt haben bürfte, weil er feine İntwort auf fein Sdareiben yon

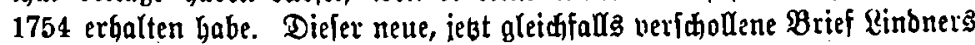
mag aus dem Spätherbft ober aus ben erften $\mathfrak{B i n t e r w o d h e n ~} 1759$ ftammen

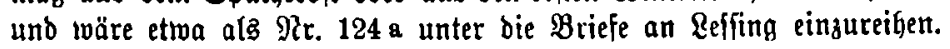

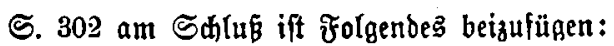

ธ. 412. Der afz verloren bezeidnete Brief $\mathfrak{M r}$. 322 an Chriftian

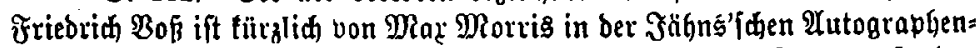

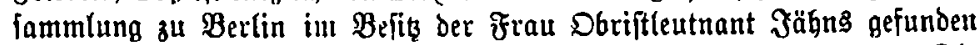
und 1914 im "Eupforion“, Bo. XX, S. 360 f. mitgeteift worben. Die bandiarift beftegt aus einem $\mathfrak{Q}$ uartblatt, bas nur auf einer Seite be= idfrieben ift; die Moreffe feght. Der Brief felbft lautet: 


\section{Riebjter Freund,}

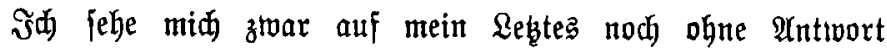

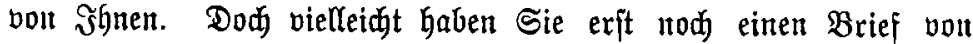
Inir erwarten rollen: ullo zwar ${ }^{1}$ ben mit bem veriprodfenen $\mathfrak{A}_{\mathfrak{n}}$, fange zu bem Bande ber Trauerfpiele. Şier ijt er, biejer $\mathfrak{A}$ nfang; nefmmlid bie ganze - jo viel id es für nötfig eradtet habe, burdicorrigirte Sara. Mädjtens foll ber Philotas forgen; und id

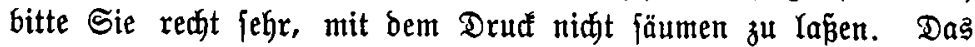

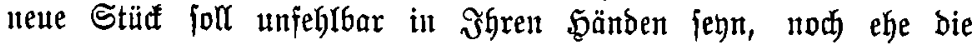
alte Materie ganz abgebruftt toorben. (Es geht alles recht gut;

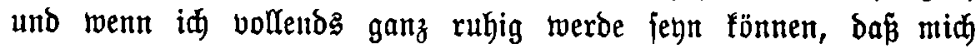
gegen Betygnadten nidft meine Sduulben wild madjen: jo wirb ez now befier geher. $3 \mathfrak{u}$ dent zwenten Bande ber Sdjriften follen Sie intuerbalb adjt Tagen ebenfalls mehr Manujcript haben - helfen Sie mir, liebjter Freund, nur nod diejen Sturm auß̧দalten: und

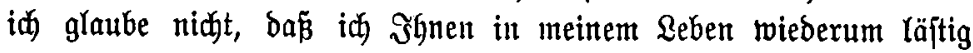
fallen will. Leben Sie inbeß recht wohy.

$$
\begin{array}{cc}
\text { Wolfenbüttel } & \text { Dero } \\
\text { bent } 1 \text { ten Xbr } 1771 & \text { ergebenfter } \mathfrak{F r} \text {. }
\end{array}
$$

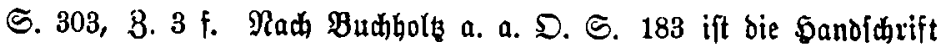

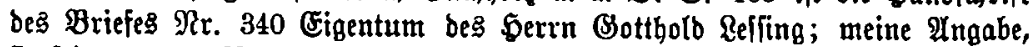

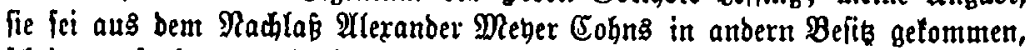

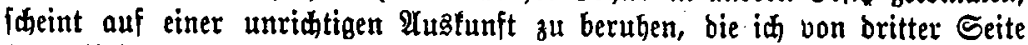
her erbielt.

S. 303-309. Die Erännzung bes stiefes an Wieland $\mathfrak{9}$ r. 429, ferner bie Briefe von Reffing 9 R. $477 \mathrm{a}, 548 \mathrm{a}, 637 \mathrm{a}$ und 783 a fino nun

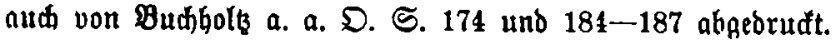

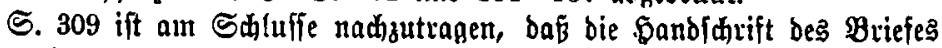

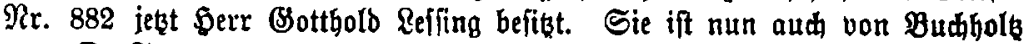
a. a. S. S. 187 abgebrudt.

ธ. 312 f. Der Brief an Refing $\mathfrak{R}$. 465 a ift jeģt ebenfalls von Buthbolis a. a. D. S. 192 abgedrưt.

S. 314. Finter 3. 6 ift einzufügen: "S. XLI [ift bei "linoner, Johann Bottbeff" nod ein Brief von Reffing zu verzeidnen:] 143 a Jan.

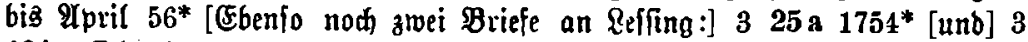
124 a Slt. bis Dez. 59*“. EGbenda ift Ginter 8.17 einzufügen: "S. LII $\uparrow$.

\footnotetext{
' unb ztwar [berbefiert aus] nebmli
} 


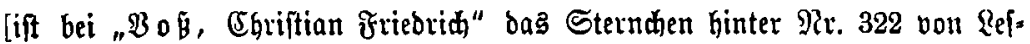
fing zu ftreiden, ferner noch ein Brief an Reffing zu verzeidnen:] 4465 a 10 Märs 72". -

Fraft nodh reider als bei ben früheren Bänden wurbe idh bei biefem

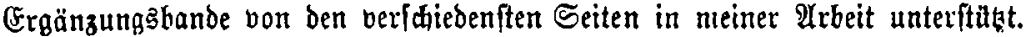

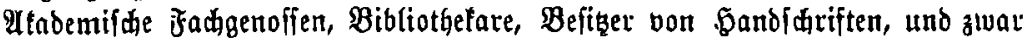
Freunbe unb längft erprobte Bsönner ebenfowohl wie Frembe, an bie id mich zun eriten Male wanbte, haben mid nut gleider Bereitmilligfeit gefösdert.

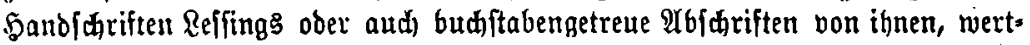
volle $\mathfrak{A} u$ sflünfte über fie jandten mir, zum Ieil von freien Stüđen, ofne nur ucine Bitte abzumarten, bie Bibliotgets: und Ardivoorftände Beorg Minde. Bouet in SBromberg, $\mathbb{S} u f t a b$ Mild

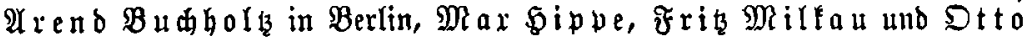

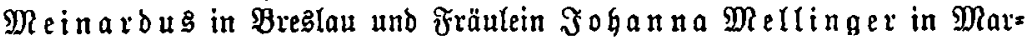
bad, bie $\mathfrak{B e r w a l t u n g}$ ber töniglidłen $\mathfrak{B i b l i o t b e t ~ i n ~ B e r l i n , ~ o e r ~ U n i v e r f i t a ̈ t s : ~}$ bibliothet in Ssöttingen, ber Stabtbibliothet in Samburg, bes Reftner=Mufeums

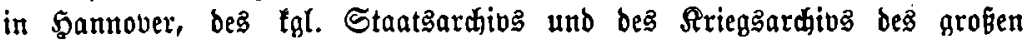

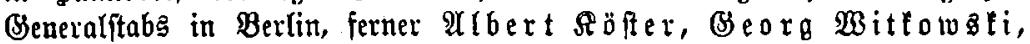

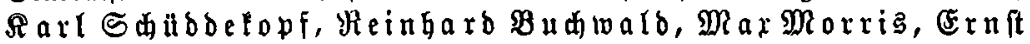

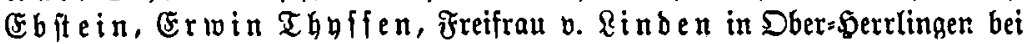

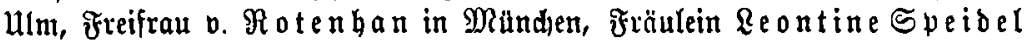

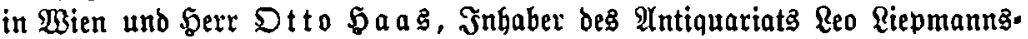
fobn in Bertin. Bana befonders zu Danl verpflidtet bin ind für foldhe

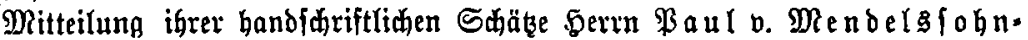
Bartholdy in Berlin und Serrn Rittergutebefizer Botthold Reffing zu Meéeberg bei Berlin, ber mir mit bemfelben, immer wieder zur silfe bes reiten Boblrwollen treu zur Seite ftand vie zubor fein nun veremigter, mir

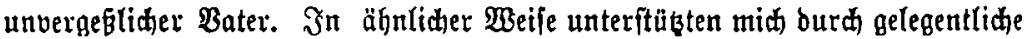

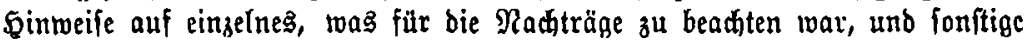
Quffalüffe Beragard Senffert, Juliug Beterfen, Stepgan

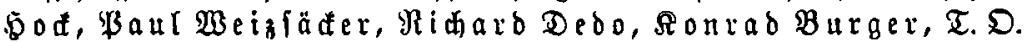

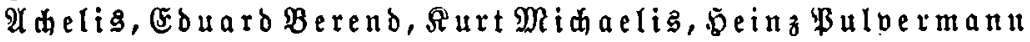
und ber, ben iđh mir gerabe für biefen Ergänzungsband gern alz erften

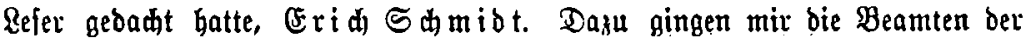

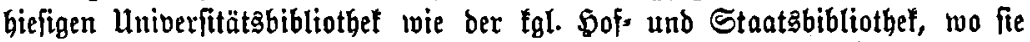
nur fonnten, eifrig an bie sand. In Gerzlither Danfbarfeit mödte ith aud bier aller bicfer freunbliden belfer gebenten.

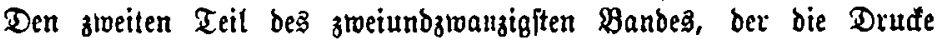

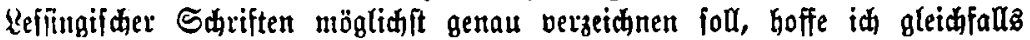

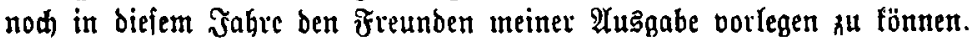

$\mathfrak{M}$ ü $\mathfrak{n}$ he $\mathfrak{n}$, in $\mathfrak{M a i} 19 \vdash 5$.

\#rani Z72undker. 